\title{
High expression of HVEM is associated with improved prognosis in intrahepatic cholangiocarcinoma
}

\author{
BINGQI MA ${ }^{1,2}$, HUIJUAN MENG ${ }^{2}$, YE TIAN $^{3}$, YINGYING WANG ${ }^{1}$, TIANQIANG SONG ${ }^{1}$, \\ TI ZHANG ${ }^{1}$, QIANG WU ${ }^{1}$, YUNLONG CUI ${ }^{1}$, HUIKAI LI $^{1}$, WEI ZHANG ${ }^{1}$ and QIANG LI ${ }^{1}$ \\ ${ }^{1}$ Department of Hepatobiliary Surgery, Tianjin Medical University Cancer Institute and Hospital; \\ National Clinical Research Center for Cancer; Key Laboratory of Cancer Prevention and Therapy, Tianjin; \\ Tianjin's Clinical Research Center for Cancer, Tianjin 300060; ${ }^{2}$ Affiliated Hospital of Weifang Medical University, \\ Weifang, Shandong 261031; ${ }^{3}$ Department of Senior Ward, Tianjin Medical University Cancer Institute and Hospital; \\ National Clinical Research Center for Cancer; Key Laboratory of Cancer Prevention and Therapy, \\ Tianjin; Tianjin's Clinical Research Center for Cancer, Tianjin 300060, P.R. China
}

Received August 10, 2020; Accepted October 29, 2020

DOI: $10.3892 / \mathrm{ol} .2020 .12330$

\begin{abstract}
Herpesvirus entry mediator (HVEM) displays dual signals in T-cell activation according to the ligands and intracytoplasmic effectors it interacts with. High HVEM expression may play an immunosuppressive role in several malignancies. The present study investigated the clinical impact of HVEM on intrahepatic cholangiocarcinoma (ICC), including its prognostic value, and association with clinicopathological features and immune status. The clinical data of 102 consecutive patients with ICC who underwent surgical treatment from January 2012 to December 2017 were collected. The expression of HVEM and different types of tumor-infiltrating lymphocytes (TILs) were investigated in ICC tissue samples by immunohistochemical staining. HVEM expression was detected in the tumor tissues of $92(90.2 \%)$ patients with ICC. Patients with high HVEM expression were more likely to
\end{abstract}

Correspondence to: Dr Wei Zhang or Dr Qiang Li, Department of Hepatobiliary Surgery, Tianjin Medical University Cancer Institute and Hospital; National Clinical Research Center for Cancer; Key Laboratory of Cancer Prevention and Therapy, Tianjin; Tianjin's Clinical Research Center for Cancer, 3 Huanhu West Road, Tianjin 300060, P.R. China

E-mail: zhangweitjch@163.com

E-mail: liqiang6011@126.com

Abbreviations: ICC, intrahepatic cholangiocarcinoma; HVEM, herpesvirus entry mediator; Mon, monocyte; TIL, tumor-infiltrating lymphocyte; DFS, disease-free survival; OS, overall survival; TMAs, tissue microarrays; IHC, immunohistochemistry; Lym, lymphocyte; HR, hazard ratio; CIs, confidence intervals; PBL, peripheral blood lymphocyte; Neu, neutrophil; NLR, neutrophil-lymphocyte ratio

Key words: intrahepatic cholangiocarcinoma, herpesvirus entry mediator, tumor-infiltrating lymphocyte, peripheral blood lymphocyte, prognosis have increased peripheral blood lymphocyte (PBL) concentrations $(\mathrm{P}=0.031)$, decreased CEA $(\mathrm{P}=0.036)$, low TNM stage $(\mathrm{P}=0.043)$ and high frequencies of small-duct histological type $(\mathrm{P}=0.021)$ and BAP1 retained expression $(\mathrm{P}=0.010)$. Survival analysis showed that high HVEM expression was a favorable independent predictor of overall postoperative survival $(\mathrm{P}=0.034$, hazard ratio $=0.486,95 \%$ confidence interval $=0.249-0.945)$. In addition, no significant association of HVEM expression with $\mathrm{CD}^{+}(\mathrm{P}=0.512), \mathrm{CD}^{+}(\mathrm{P}=0.750)$ or $\mathrm{CD}_{45 \mathrm{RO}^{+}}(\mathrm{P}=0.078)$ TILs was identified in the ICC tissues. These results indicate that HVEM may serve as a favorable prognostic marker for ICC. Furthermore, co-stimulatory signals from HVEM may play a dominant role in the progression of ICCs, which can be explained by an increase in the number of PBLs rather than a change in the number of TILs. However, the function of the HVEM network in ICC progression is complex and requires further study.

\section{Introduction}

Intrahepatic cholangiocarcinoma (ICC) is the second most common primary hepatic malignant tumor after hepatocellular carcinoma (HCC) and its age-adjusted incidence rate increased by almost $20 \%$ from 0.75 per 100,000 in 1992 to 0.88 per 100,000 in 1999 (1). It is a highly fatal malignancy with a median postoperative survival time of 28 months (2). Once ICC has become unresectable, the median survival time is only 10 months (3). The main treatments for ICC include surgery, locoregional therapy and systemic chemotherapy. However, the efficacy of locoregional therapy for advanced or unresectable ICC is limited (4,5). A meta-analysis (2) showed that patients with ICC did not benefit from systemic chemotherapy based on gemcitabine, fluorouracil or oxaliplatin. Furthermore, the marked heterogeneity of ICC has led to a lack of effective targeted agents for the treatment of this fatal disease (5). Consequently, new drugs or therapeutic strategies are urgently required to improve the survival prognosis of patients with ICC. Immunotherapy is a rapidly evolving 
therapeutic strategy in oncology. The immune system has the potential to recognise and eradicate cancer cells, and is regulated by a complex network of immune checkpoints. Cancers are able to evade host antitumor immune responses through immune escape mechanisms. Immune checkpoint inhibitors, including antibodies against programmed cell death protein 1 (PD-1)/programmed death ligand 1orcytotoxic T-lymphocyte-associated protein 4 , interrupt the mechanisms of immune resistance and exhibit durable and powerful antitumor activity in specific subsets of patients across several types of tumor (6).

Herpesvirus entry mediator (HVEM), also known as tumor necrosis factor receptor superfamily 14 , is widely expressed on a range of hematopoietic cells, including B cells, T cells, NK cells, monocytes and immature dendritic cells, and several non-hematopoietic cells and tissues, including the liver, kidney and lung $(7,8)$. HVEM is a ligand for cytokines of the TNF superfamily, including lymphotoxin $\alpha$ and lymphotoxin-related inducible ligand that competes for glycoprotein D binding to HVEM on T cells (LIGHT), or a receptor for members of the immunoglobulin superfamily, including CD160 and B- and T-lymphocyte attenuator (BTLA), under diverse physiological and pathological conditions. Interactions of HVEM with different family members occur at distinct sites, and result in some opposing functions. When HVEM expressed by antigen-presenting cells interacts with TNF superfamily cytokines, the activation of T-cell proliferation and cytokine production is triggered. Conversely, the binding of HVEM to CD160 or BTLA results in inhibitory signaling to $\mathrm{T}$ cells (9). However, Ritthipichai et al (10) reported that BTLA harnesses cytosolic adaptor growth factor receptor-bound protein 2 (GRB2) to provide co-stimulatory signals to $\mathrm{CD} 8^{+} \mathrm{T}$ cells. Therefore, it appears that the HVEM/BTLA pathway plays a dual function in T-cell activation.

In addition to normal hematopoietic and non-hematopoietic cells, HVEM expression is detected in most cancer cells, including pancreatic and ampullary cancer (11), HCC (12), oesophageal squamous cell carcinoma (13), gastric cancer (14), clear cell renal cell carcinoma (15), ovarian cancer $(16,17)$, breast cancer (18) and melanoma (19). In the majority of cases, the high expression of HVEM is associated with poor prognosis (12-19) and is associated with low levels of tumor-infiltrating lymphocytes (TILs) and downregulation of the local immune response $(12,13,18)$. These previous studies suggest that HVEM is potentially an independent prognostic marker in patients with specific cancers and a potential target for antitumor therapy. However, the precise function of HVEM in ICC has rarely been studied. The present study aimed to investigate the clinical impact of HVEM in ICC, including its prognostic value and association with clinicopathological features and immune status.

\section{Materials and methods}

Patients and tissue samples. A total of 102 consecutive patients with ICC who underwent surgical treatment at Tianjin Medical University Cancer Institute and Hospital from January 2012 to December 2017 were evaluated in the present study. Cases with combined HCC-CCA, composed of typical
HCC and representative ICC, were excluded. In addition, patients who had undergone preoperative treatments, such as radiofrequency ablation, adjuvant chemotherapy or radiation therapy, were excluded. All patients were reviewed to confirm the diagnosis of ICC and to determine the stage according to the American Joint Committee on Cancer staging system (8th edition, 2017). Baseline clinicopathological features and information were reviewed and analyzed. All cases were regularly followed up every 3 months to determine whether tumor recurrence occurred. Disease-free survival (DFS) was measured from the date of surgery to the date of first recurrence or last follow-up, whereas overall survival (OS) was defined as the interval between the date of first diagnosis of ICC and the date of death or last follow-up.

Formalin-fixed paraffin-embedded tissues and hematoxylin-eosin stained slides from the 102 cases of ICC were collected. Tissue microarrays (TMAs) with a thickness of 4- $\mu \mathrm{m}$ consisting of 2-mm cores of tumor samples were constructed by selecting a typical tumor region and a representative peritumoral area from each case.

The Medical Ethics Committee of Tianjin Medical University Cancer Institute and Hospital approved the present study (approval no. bc2019065), and written informed consent was obtained from all patients or their legal guardian.

Immunohistochemistry (IHC). TMA slides were heated at $65^{\circ} \mathrm{C}$ for $2 \mathrm{~h}$, routinely dewaxed in xylene and rehydrated in gradient ethanol. After antigen retrieval, the slides were blocked with $3 \%$ hydrogen peroxide (PV-6002; Beijing Zhongshan Golden Bridge Biotechnology Co., Ltd.) for $10 \mathrm{~min}$ at room temperature to quench the endogenous peroxidase activity. The sections were incubated with primary antibodies at $4^{\circ} \mathrm{C}$ overnight and $37^{\circ} \mathrm{C}$ for $1 \mathrm{~h}$, followed by HRP-conjugated secondary antibody at $37^{\circ} \mathrm{C}$ for $1 \mathrm{~h}$. Then, the sections were visualised with 3,3'-diaminobenzidine (ZLI-9017; OriGene Technologies) for $5 \mathrm{~min}$ at room temperature and counterstained with hematoxylin for $1 \mathrm{~min}$ at room temperature. Appropriate positive (formalin-fixed paraffin-embedded sections of human lung tissue) and negative controls were designed and used for each round. The sections were observed and recorded using a light microscope (BX61, Olympus ).

The primary antibodies used in the present study were as follows: HVEM/TNFRSF14 (MAB3561; concentration, $16 \mu \mathrm{g} / \mathrm{ml}$; R\&D Systems, Inc.), CD4 (ab133616; dilution, 1:500; Abcam), CD8 (ab17147; dilution, 1:50; Abcam) andCD45RO (sc-1183; dilution, 1:500; Santa Cruz Biotechnology, Inc.).

The staining pattern revealed that HVEM was expressed in the cytoplasm and cell membrane. The staining was evaluated semiquantitatively by multiplying scores for the intensity and percentage of positive tumor cells; representative images are presented in Fig. 1. The intensity of staining was divided into four subgroups: Score 0, negative; score 1, mild; score 2, moderate; and score 3, strong. The percentage of staining was determined as follows: Score $0,<1 \%$; score 1, $1-25 \%$; score 2, 26-50\%; score 3, 51-75\% and score 4, 76-100\% (17). Total scores of $\leq 6$ and $\geq 8$ were defined as low and high HVEM expression, respectively.

In the case of lymphocyte subset staining, the numbers of $\mathrm{CD}^{+}, \mathrm{CD}^{+}$and $\mathrm{CD} 45 \mathrm{RO}^{+} \mathrm{T}$ cells that infiltrated into the ICC tissues were counted manually. Four randomly selected 

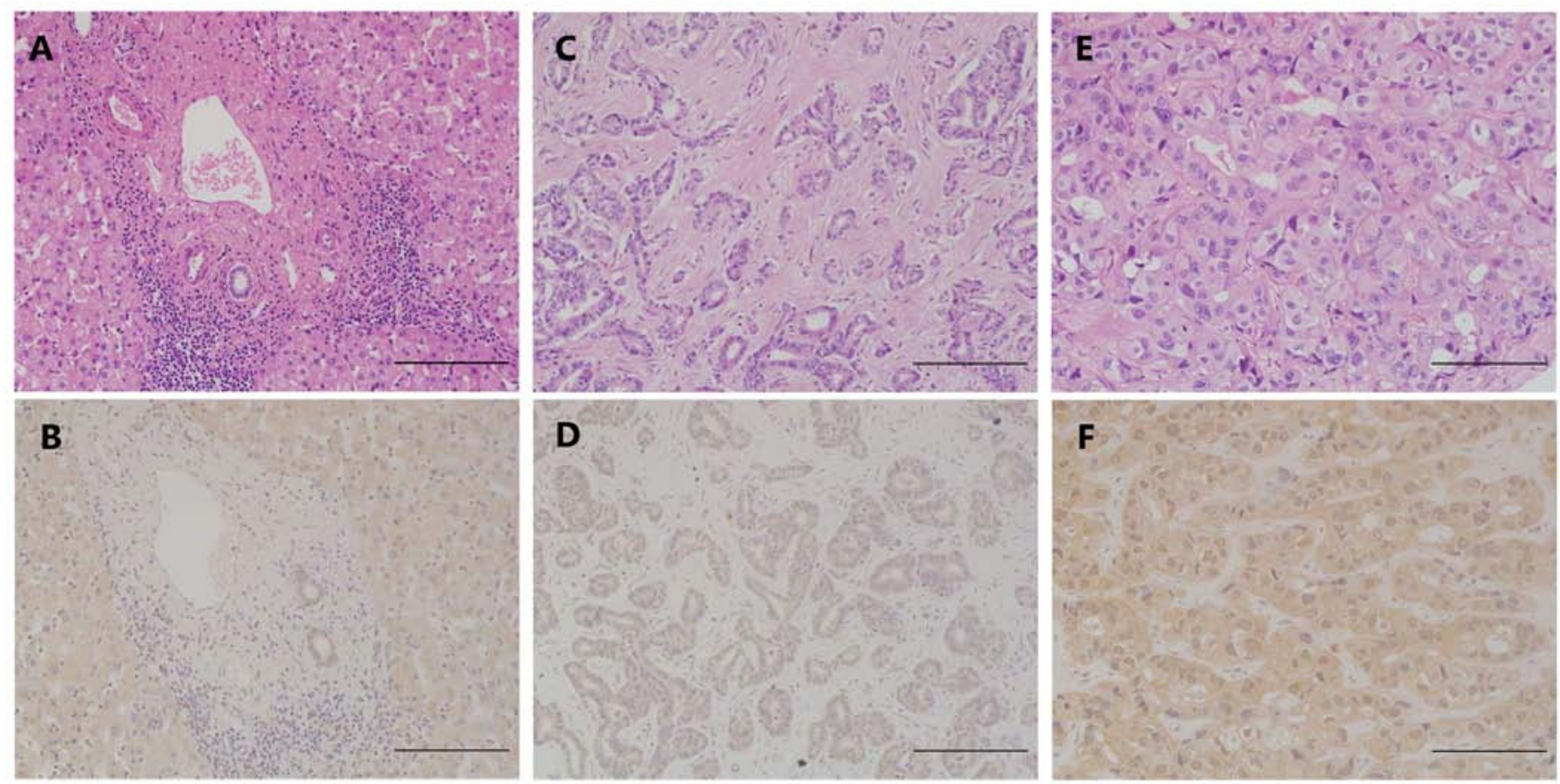

Figure 1. Representative images of HVEM immunohistochemical staining and the corresponding histology with hematoxylin-eosin staining in ICC tissues and adjacent liver tissues. (A) Histology and (B) mild-to-moderate staining of HVEM in peritumoral liver tissues. (C) Histology and (D) low expression of HVEM in ICC tissues. (E) histology and (F) high expression of HVEM in ICC tissues. Scale bar, $200 \mu \mathrm{m}$. HVEM, herpesvirus entry mediator; ICC, intrahepatic cholangiocarcinoma.

areas were counted for each sample at a magnification of $\mathrm{x} 200$ (Fig. 2). The average value was recorded for each sample.

All IHC scoring was performed independently by two investigators without knowledge of the clinical data. Final agreement on the scores was reached through full discussion.

Analysis of histological classification and frequent mutations in ICC. Our previous study (20) classified this cohort into two subtypes, namely large-duct and small-duct types with regard to histological characteristics, S100P expression and Alcian blue scores (Data S1). The number of cases identified as large-duct and small-duct types of ICC were 21 (20.6\%) and $81(79.4 \%)$ respectively. Frequently mutated genes in ICC, including isocitrate dehydrogenase 1/2 (IDH1/2), BRCA1 associated protein 1 (BAP1), AT-rich interaction domain 1A (ARID1A) and polybromo 1 (PBRM1) were also analyzed (Data S1). DNA sequencing showed that 17 cases (16.7\%) harboured IDH1/2 mutations, whereas IHC analysis showed the loss of expression of BAP1, ARID1A and PBRM1 in $46(45.1 \%), 20(19.6 \%)$ and $33(32.4 \%)$ of cases, respectively.

Statistical analysis. Categorical variables (clinicopathological factors) are presented as total numbers and frequencies and were evaluated by Chi-square test. Continuous variables (number of TILs) are presented as medians with ranges and were compared using Mann-Whitney U test. Survival analysis was calculated by the Kaplan-Meier method and assessed by log-rank test for univariate analysis. Cox proportional hazards model was used for analyzing the prognostic value of HVEM expression and other clinical factors. The results are presented as the hazard ratio (HR) and $95 \%$ confidence intervals (CIs). A two-tailed $\mathrm{P}<0.05$ was considered to indicate a statistically significant difference. SPSS Statistics 24 (IBM Corp.) was used for data analysis.

\section{Results}

HVEM expression in ICC and adjacent liver tissues. HVEM expression in the ICC tissues exhibited significant heterogeneity. No HVEM staining was detected in the tumor tissues of 10 cases $(9.8 \%)$, whereas the tumor tissues of the other cases exhibited mild-to-strong staining. According to the aforementioned classification criteria, low and high HVEM expression was detected in 50 and 52 of the 102 specimens, respectively. In addition, the peritumoral liver tissues in all cases presented mild-to-moderate staining of HVEM (Fig. 1, Table I).

Association between HVEM expression and clinicopathological characteristics in patients with ICC. The study cohort included 57 males (55.9\%) and 45 females (44.1\%), with a mean age of $57.7 \pm 9.4$ years (range, 28-77 years). Fifty-five cases (53.9\%) underwent lymphadenectomy.

Patients with high HVEM expression had an increased peripheral blood lymphocyte (PBL) concentration $(\mathrm{P}=0.031)$, decreased CEA concentration $(\mathrm{P}=0.036)$, low TNM stage $(\mathrm{P}=0.043)$ and high frequencies of small-duct histological subtype $(\mathrm{P}=0.021)$ and $\mathrm{BAP} 1$ retained expression $(\mathrm{P}=0.010)$ (Table I). The association between HVEM expression and PBL concentration indicated that HVEM expression might enhance the immune response by increasing the number of PBLs in patients with intrahepatic cholangiocarcinoma.

Prognostic significance of HVEM in patients with ICC. Survival analysis revealed 3-year DFS and OS rates of 
Table I. Association between HVEM expression and clinicopathological characteristics in 102 patients with intrahepatic cholangiocarcinoma.

\begin{tabular}{|c|c|c|c|}
\hline \multirow[b]{2}{*}{ Variables } & \multicolumn{2}{|c|}{ HVEM expression } & \multirow[b]{2}{*}{ P-valu } \\
\hline & Low & High & \\
\hline Sex & & & 0.439 \\
\hline Male & $26(45.6)$ & $31(54.4)$ & \\
\hline Female & $24(53.3)$ & $21(46.7)$ & \\
\hline Age (years) & & & 0.411 \\
\hline$\leq 60$ & $30(52.6)$ & $27(47.4)$ & \\
\hline$>60$ & $20(44.4)$ & $25(55.6)$ & \\
\hline Hepatitis B & & & 0.397 \\
\hline Negative & $32(52.5)$ & $29(47.5)$ & \\
\hline Positive & $18(43.9)$ & $23(56.1)$ & \\
\hline Neu $\left(\times 10^{9} / 1\right)$ & & & 0.723 \\
\hline$\leq 5.0$ & $41(48.2)$ & $44(51.8)$ & \\
\hline$>5.0$ & $9(52.9)$ & $8(47.1)$ & \\
\hline $\operatorname{Lym}\left(x 10^{9} / 1\right)$ & & & 0.031 \\
\hline$\leq 2.0$ & $38(56.7)$ & $29(43.3)$ & \\
\hline$>2.0$ & $12(34.3)$ & $23(65.7)$ & \\
\hline Mon $\left(\times 10^{9} / 1\right)$ & & & 0.727 \\
\hline$\leq 0.6$ & $33(47.8)$ & $36(52.2)$ & \\
\hline$>0.6$ & $17(51.5)$ & $16(48.5)$ & \\
\hline $\mathrm{CEA}(\mu \mathrm{g} / \mathrm{l})$ & & & 0.036 \\
\hline$\leq 5$ & $36(43.9)$ & $46(56.1)$ & \\
\hline$>5$ & $14(70.0)$ & $6(30.0)$ & \\
\hline CA19-9 (U/ml) & & & 0.570 \\
\hline$\leq 39$ & $28(46.7)$ & $32(53.3)$ & \\
\hline$>39$ & $22(52.4)$ & $20(47.6)$ & \\
\hline Histological grade & & & 0.868 \\
\hline G1-G2 & $29(48.3)$ & $31(51.7)$ & \\
\hline $\mathrm{G} 3$ & $21(50.0)$ & $21(50.0)$ & \\
\hline $\mathrm{T}$ category & & & 0.193 \\
\hline $\mathrm{T} 1-\mathrm{T} 2$ & $42(46.7)$ & $48(53.3)$ & \\
\hline T3-T4 & $8(66.7)$ & $4(33.3)$ & \\
\hline $\mathrm{N}$ category & & & 0.141 \\
\hline NO & $19(51.4)$ & $18(48.6)$ & \\
\hline N1 & $13(72.2)$ & $5(27.8)$ & \\
\hline M category & & & 0.156 \\
\hline M0 & $44(46.8)$ & $50(53.2)$ & \\
\hline M1 & $6(75.0)$ & $2(25.0)$ & \\
\hline TNM stage & & & 0.043 \\
\hline I-II & $16(48.5)$ & $17(51.5)$ & \\
\hline III-IV & $16(76.2)$ & $5(23.8)$ & \\
\hline Histological subtype & & & 0.021 \\
\hline Large-duct & $15(71.4)$ & $6(28.6)$ & \\
\hline Small-duct & $35(43.2)$ & $46(56.8)$ & \\
\hline IDH1/2 & & & 0.479 \\
\hline Wild type & $43(50.6)$ & $42(49.4)$ & \\
\hline Mutant & $7(41.2)$ & $10(58.8)$ & \\
\hline BAP1 & & & 0.010 \\
\hline Lost & $29(63.0)$ & $17(37.0)$ & \\
\hline Retained & $21(37.5)$ & $35(62.5)$ & \\
\hline
\end{tabular}

Table I. Continued.

\begin{tabular}{lccc}
\hline & \multicolumn{2}{c}{ HVEM expression } & \\
\cline { 2 - 3 } Variables & Low & High & P-value \\
\hline Sex & & & 0.439 \\
IRID1A & & & 0.273 \\
$\quad$ Lost & $12(60.0)$ & $8(40.0)$ & \\
Retained & $38(46.3)$ & $44(53.7)$ & \\
PBRM1 & & & 0.727 \\
$\quad$ Lost & $17(51.5)$ & $16(48.5)$ & \\
Retained & $33(47.8)$ & $36(52.2)$ & \\
\end{tabular}

Values are presented as n (\%). HVEM, herpesvirus entry mediator; Neu, neutrophil; Lym, lymphocyte; Mon, monocyte; IDH1/2, isocitrate dehydrogenase $1 / 2$; BAP1, BRCA1 associated protein 1 ; IRID1A, AT-rich interaction domain 1A; PBRM1, polybromo 1.

30.4 and $59.4 \%$, respectively. The median survival time was 42.9 months, and the median follow-up period was 25.1 months (range, 4.9-100.0 months).

Univariate analysis showed that patients with ICC and high HVEM expression had significantly improved the survival DFS time $(\mathrm{P}=0.008)$ and $\mathrm{OS}(\mathrm{P}=0.005)$ than patients with low HVEM expression. In addition, the neutrophil-lymphocyte ratio, CA19-9 concentration, $\mathrm{T}$ category and $\mathrm{M}$ category were prognostic factors for DFS and OS in ICC $(\mathrm{P}<0.020)$. Multivariate analysis included these five factors with $\mathrm{P}<0.02$, and revealed that high HVEM expression was a favorable independent predictor of $\mathrm{OS}(\mathrm{P}=0.034, \mathrm{HR}=0.486$, 95\% $\mathrm{CI}=0.249-0.945)$ in ICC. However, HVEM expression only tended to be an independent prognostic factor for DFS $(\mathrm{P}=0.054, \mathrm{HR}=0.604,95 \% \mathrm{CI}=0.361-1.009)$ in $\mathrm{ICC}$, because the association with DFS did not reach statistical significance (Fig. 3, Table II).

Association of HVEM expression with TILs in ICC. TILs were investigated by IHC to explore the underlying mechanism of the predictive value of HVEM expression in ICC. The number of $\mathrm{CD}^{+}, \mathrm{CD}^{+}$and $\mathrm{CD} 45 \mathrm{RO}^{+}$TILs in different ICC tissue samples varied significantly and did not follow a normal distribution. The results revealed that HVEM expression was not significantly associated with $\mathrm{CD}^{+}(\mathrm{P}=0.512)$, $\mathrm{CD}^{+}(\mathrm{P}=0.750)$ or $\mathrm{CD}^{2} \mathrm{RO}^{+}(\mathrm{P}=0.078)$ TILs (Fig. 2, Table III). The results indicate that HVEM expression has no significant effect on the infiltration of T-cell subsets into ICC tissues.

Prognostic value of PBL and TIL subsets in ICC. Survival analysis demonstrated that patients with high PBL concentration shad a significantly prolonged OS time $(\mathrm{P}=0.048)$ and an increased DFS time that was not statistically significant $(\mathrm{P}=0.097)$ in ICC (Fig. S1). Meanwhile, $\mathrm{CD}^{+}$, $\mathrm{CD}^{+}$and $\mathrm{CD} 45 \mathrm{RO}^{+}$TILs were not significantly associated with DFS $(\mathrm{P}=0.934, \mathrm{P}=0.717$ and $\mathrm{P}=0.816$, respectively) or $\mathrm{OS}$ $(\mathrm{P}=0.958, \mathrm{P}=0.485$ and $\mathrm{P}=0.416$, respectively) in patients with ICC (Figs. S2-S4). 

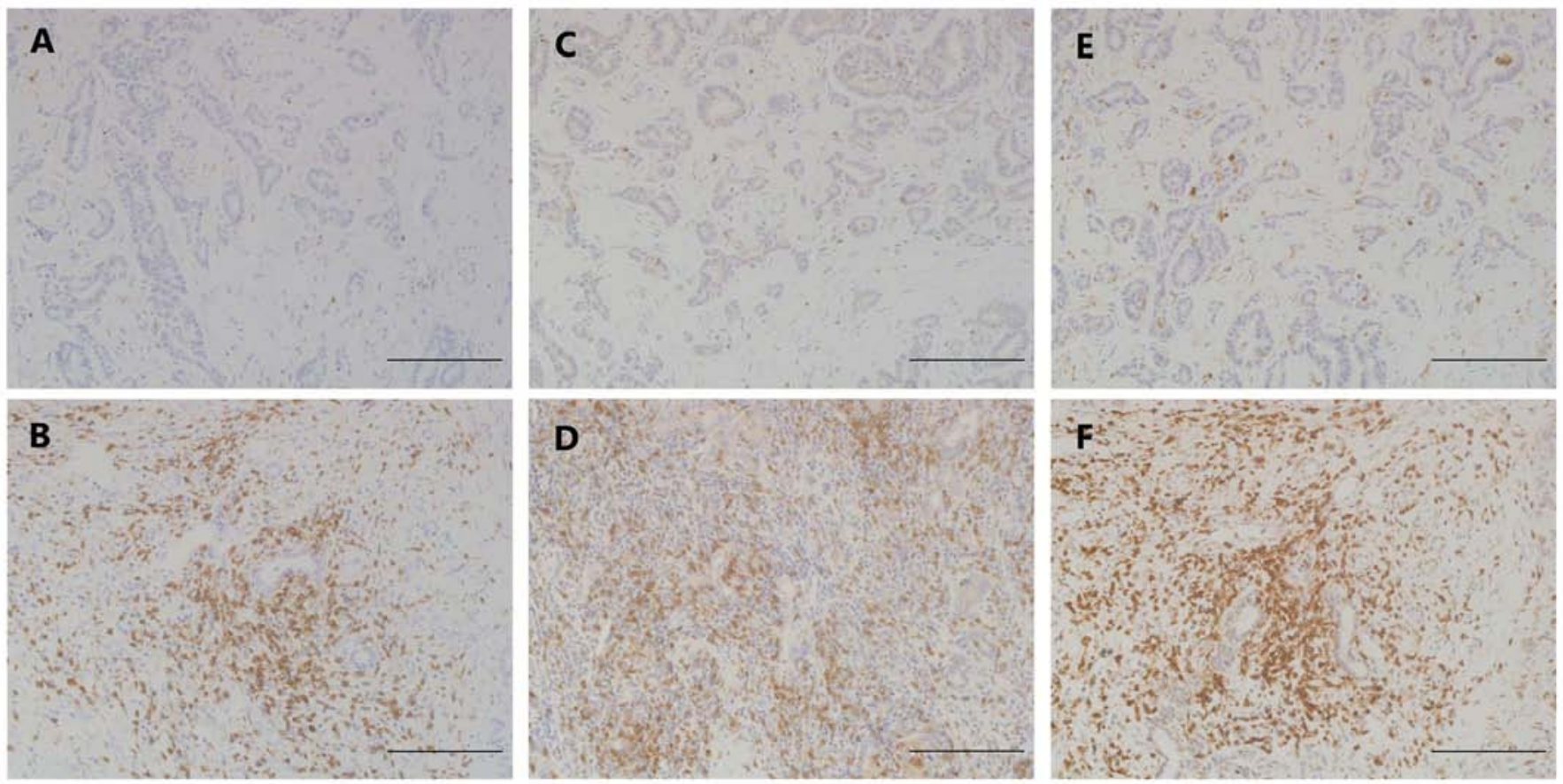

Figure 2. Representative images of immunohistochemical staining of TIL subsets in intrahepatic cholangiocarcinoma. (A) Low and (B) high levels of CD4 ${ }^{+}$ TILs. (C) Low and (D) high levels of CD8 $8^{+}$TILs. (E) Low and (F) high levels of CD45RO ${ }^{+}$TILs. Scale bar, $200 \mu$ m. TIL, tumor-infiltrating lymphocyte; HVEM, herpesvirus entry mediator.

A

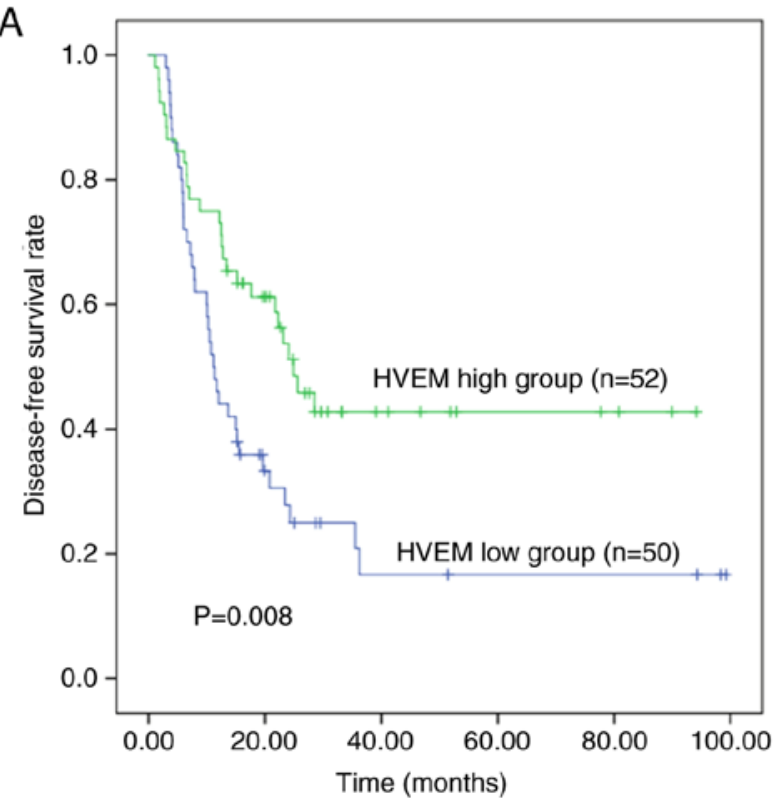

B

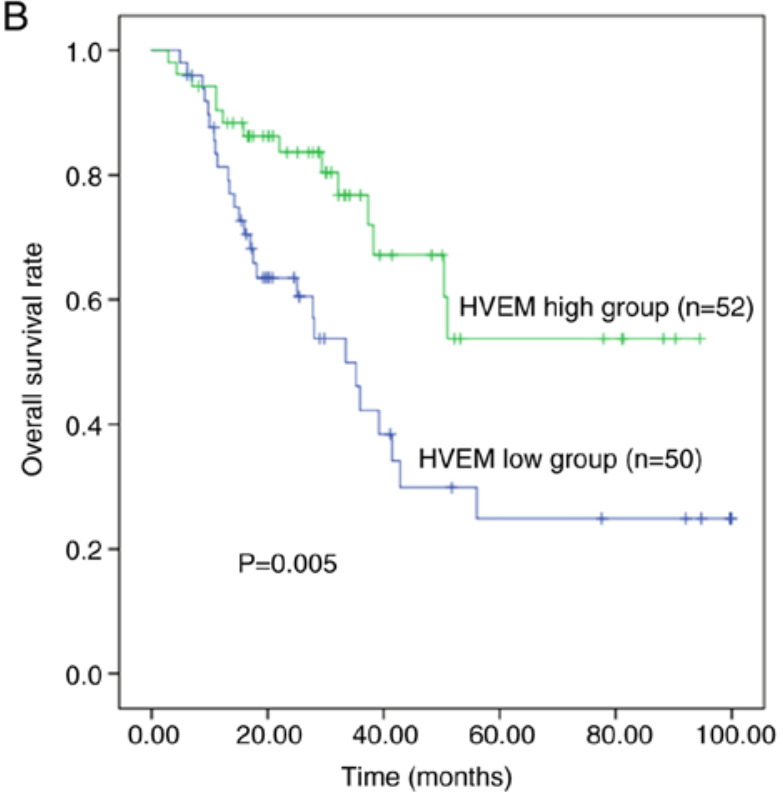

Figure 3. Kaplan-Meier curves for patients within intrahepatic cholangiocarcinoma. Patients with high expression of HVEM had prolonged (A) disease-free survival and (B) overall survival. HVEM, herpesvirus entry mediator.

\section{Discussion}

Immunotherapeutic strategies aim to enhance the host antitumor immune response and restrain the ability of tumors to evade that response by blocking co-inhibitory signals. Given the limited efficacy of the PD-1-targeting antibody pembrolizumab in the treatment of biliary tract cancer in clinical trials (21), targeting HVEM could be a complementary or alternative strategy to improve the outcome of patients with ICC. However, it is necessary to determine the expression status of HVEM and its association with clinical outcome in patients with ICC in order to support this suggestion.

Firstly, the present study confirmed that HVEM was expressed in $90.2 \%$ of 102 ICC specimens using IHC, and found that high HVEM expression was a favorable independent predictor of postoperative survival and associated with a trend towards low $\mathrm{CD} 45 \mathrm{RO}^{+}$TIL concentrations in patients with ICC. These data are consistent with a recent study by 
Table II. Univariate and multivariate analysis for prognostic factors in intrahepatic cholangiocarcinoma.

\begin{tabular}{|c|c|c|c|c|c|c|c|c|}
\hline \multirow[b]{3}{*}{ Variables } & \multicolumn{4}{|c|}{ DFS } & \multicolumn{4}{|c|}{ OS } \\
\hline & \multirow{2}{*}{$\begin{array}{l}\text { Univariate } \\
\text { P-value }\end{array}$} & \multicolumn{3}{|c|}{ Multivariate } & \multirow{2}{*}{$\begin{array}{c}\text { Univariate } \\
\text { P-value }\end{array}$} & \multicolumn{3}{|c|}{ Multivariate } \\
\hline & & HR & $95 \% \mathrm{CI}$ & P-value & & HR & $95 \% \mathrm{CI}$ & $\mathrm{P}$-value \\
\hline NLR,$\leq 3$ vs. $>3$ & 0.012 & 1.380 & $0.699-2.727$ & 0.353 & 0.004 & 1.798 & $0.785-4.115$ & 0.165 \\
\hline CA19-9, $\leq 40$ vs. $>40 \mathrm{U} / \mathrm{ml}$ & 0.017 & 1.446 & $0.857-2.441$ & 0.167 & 0.019 & 1.549 & $0.794-3.022$ & 0.200 \\
\hline $\mathrm{T}$ category, T1-T2 vs. T3-T4 & 0.013 & 1.628 & $0.757-3.500$ & 0.212 & 0.000 & 2.707 & $1.155-6.344$ & 0.022 \\
\hline M category, M0 vs. M1 & 0.000 & 2.601 & $1.054-6.419$ & 0.038 & 0.009 & 1.281 & $0.453-3.620$ & 0.641 \\
\hline HVEM expression, low vs. high & 0.008 & 0.604 & $0.361-1.009$ & 0.054 & 0.005 & 0.486 & $0.249-0.945$ & 0.034 \\
\hline
\end{tabular}

DFS, disease-free survival; OS, overall survival; NLR, neutrophil-lymphocyte ratio; HVEM, herpesvirus entry mediator.

Table III. Association between tumor HVEM expression and TILs in intrahepatic cholangiocarcinoma.

\begin{tabular}{|c|c|c|c|}
\hline \multirow[b]{2}{*}{ Variables } & \multicolumn{2}{|c|}{ HVEM expression } & \multirow[b]{2}{*}{ P-value } \\
\hline & Low & High & \\
\hline CD4+ TILs & $55(1-1,196)$ & $44(2-842)$ & 0.512 \\
\hline CD8+ TILs & $20(1-573)$ & $17(1-599)$ & 0.750 \\
\hline CD45RO+ TILs & $58(3-782)$ & $36(2-774)$ & 0.078 \\
\hline
\end{tabular}

Values are presented as median (range). HVEM, herpesvirus entry mediator; TIL, tumor-infiltrating lymphocyte.

Sideras et al (11), who showed that high tumor expression of HVEM $(\mathrm{P}=0.001)$ and a high $\mathrm{CD} 8 / \mathrm{FoxP} 3$ TIL ratio $(\mathrm{P}=0.006)$ were significantly associated with improved cancer-specific survival in patients with pancreatic and ampullary cancer. In another study, a bioinformatic analysis using The Cancer Genome Atlas database confirmed that the expression of HVEM mRNA was positively associated with OS in bladder cancer (22). However, these data are inconsistent with results obtained in previous studies on other tumor types (12-19). Thus, the function of HVEM in tumors is complex and may be tumor-type specific. Notably, HVEM is an immune checkpoint molecule that provides bidirectional signals in T-cell activation based on the ligands and intracytoplasmic effectors it interacts with. A previous study suggested that the co-stimulatory HVEM/LIGHT signaling pathway facilitates the activation of tumor-specific $\mathrm{T}$ cells, leading to the eradication of tumors (23). BTLA is predominantly located in tumor-specific T cells, and its binding to HVEM in tumor cells suppresses the response of tumor-specific $\mathrm{CD}^{+} \mathrm{T}$ cells, resulting in tumor immune evasion (9). BTLA also provides co-stimulatory signals in $\mathrm{CD}^{+} \mathrm{T}$ cells by harnessing the cytosolic adaptor GRB2 (10). However, the immunosuppressive function of HVEM appears to be predominantly effective in carcinoma. In several other malignancies, the high expression of HVEM has been found to be associated with low levels of tumor-infiltrating $\mathrm{T}$ cells and the decreased expression of interferon- $\gamma$, perforin and granzyme B $(12,13,18)$. HVEM gene silencing can significantly inhibit the proliferation and growth of tumor cells by inducing $\mathrm{CD}^{+}$cells and upregulating the local immune response in esophageal squamous cell carcinoma (13). Knockdown of HVEM has been shown to increase the sensitivity of activated $T$ cells in ovarian cancer $(13,16)$. In addition to having a modulating effect on immune function, HVEM may act directly on tumor cells. In oesophageal cancer (13) and clear cell renal cell carcinoma (15), HVEM gene silencing was shown to significantly reduce cell proliferation activity in vitro and in vivo. Thus, HVEM may drive tumor development via multiple pathways. However, the results of the present study of ICC do not appear to support these theories that high expression of HVEM can inhibit the immune function of $\mathrm{T}$ cells, resulting in tumor development. In the present study, high HVEM expression was associated with decreased CEA levels $(\mathrm{P}=0.036)$, low TNM stage $(\mathrm{P}=0.043)$ and improved survival outcome $(\mathrm{P}<0.01)$. This may be because, firstly the co-stimulatory signals from HVEM play a dominant role in the progression of ICC. In a study of melanoma, the tumor expression of HVEM was shown to be positively associated with the expression of BTLA on TILs by co-immunofluorescence analysis (19). Therefore, HVEM/BTLA may inhibit tumor progression primarily by interacting with the cytoplasmic adapter GRB2 to stimulate T-cell activation in ICC. Furthermore, increased immune responses may occur due to the binding of HVEM with LIGHT or other checkpoint regulators that have not yet been confirmed in patients with ICC. Secondly, mutation of HVEM in the extracellular or cytoplasmic domain may prevent it from binding to its ligands or influence the recruitment of adaptor protein (9), resulting in distinct roles of HVEM expression in different types of tumors. The HVEM signaling network in cancer remains unknown. Given the evident genetic and histological heterogeneity of ICC, further study is required to reveal the precise signaling pathways in which HVEM participates, and determine whether HVEM may be an effective therapeutic target in ICC.

The immune activity of HVEM can be indirectly demonstrated by the numbers of PBLs and TILs, which are closely associated with patient survival in solid tumors. Previous studies showed a significant association of high HVEM expression with low levels of $\mathrm{CD}^{+}, \mathrm{CD}^{+}$and $\mathrm{CD} 45 \mathrm{RO}^{+} \mathrm{TIL}$ and poor prognosis in $\mathrm{HCC}$ (12) and esophageal squamous cell carcinoma (13). Tsang et al (18) observed that outcomes were 
poor in patients with breast cancer with low levels of TIL and HVEM-positive tumors. These findings indicate that HVEM suppresses TILs and promotes tumor progression. However, the present study found that HVEM expression was not significantly associated with $\mathrm{CD}^{+}(\mathrm{P}=0.512), \mathrm{CD}^{+}(\mathrm{P}=0.750)$ or $\mathrm{CD}_{45 \mathrm{RO}^{+}}(\mathrm{P}=0.078)$ TILs, indicating that HVEM expression has no significant effect on the infiltration of T-cell subsets into ICC tissues. In addition, these findings do not support the suggestion that HVEM is a favorable prognostic marker for ICC. Therefore, the prognostic value of these TIL subsets was further determined and the results revealed that $\mathrm{CD} 4^{+}, \mathrm{CD} 8^{+}$ and $\mathrm{CD}_{45 \mathrm{RO}^{+}}$TILs were not significantly associated with DFS or OS in patients with ICC $(\mathrm{P}>0.05)$. Furthermore, the effect of HVEM on PBLs was investigated, and it was found that high HVEM expression was associated with an increased PBL concentration $(\mathrm{P}=0.031)$. The association between PBL concentration and prognosis was further investigated, and survival analysis showed that patients with high PBL concentration shad a significantly prolonged OS $(\mathrm{P}=0.048)$ and an increased DFS that was not statistically significant $(\mathrm{P}=0.097)$ in ICC. Thus, it appears that the role of HVEM involves the activation of tumor immunity by increasing the number of PBLs in patients with ICC. This finding indicates that HVEM may be a favorable prognostic marker for ICC, although it also appears to be inconsistent with results obtained in previous studies on HCC. In one study, the levels of membrane and soluble HVEM were downregulated and upregulated on PBLs, respectively, and the latter was positively associated with advanced tumor stages, suggesting that the role of HVEM maybe immunosuppressive (24). Furthermore, in another study, blockade of the BTLA/HVEM pathway increased IFN $-\gamma$ production in the circulating $\mathrm{T}$ cells of patients with HCC (25).

In general, PBLs should infiltrate into the tumor microenvironment at an early stage of tumor progression to play a part in immune surveillance. Therefore, elevated PBL and TILs should be associated with improved prognosis $(9,10,13,18)$, which our studies in ICC did not support. However, tumor signaling pathways are complex, andthe antitumor effect of HVEM in ICC may be achieved through other signaling pathways, rather than by direct alteration of the infiltration of lymphocytes. Alternatively, an association of TIL subsets with HVEM expression and prognosis may exist in ICC; however, the association did not reach statistical significance in the present study, which may be due to the small sample size. The HVEM network is complicated, and its effect on TILs and PBLs in ICC requires further study.

The association of HVEM expression with several clinicopathological characteristics was investigated in the present study, and it was revealed for the first time, to the best of our knowledge, that HVEM expression is positively associated with the frequencies of small-duct type $(\mathrm{P}=0.021)$ and BAP1 retained expression $(\mathrm{P}=0.010)$ in ICC. ICCs are heterogeneous tumors that can be histologically subdivided into two types based on the size of the biliary duct, namely small-duct and large-duct types (26). In addition, this classification individuates ICC subgroups with different clinicopathological and molecular characteristics $(26,27)$. Therefore, high HVEM expression appears to be a specific feature of small-duct type ICC. BAP1 is a commonly mutated gene involved in histone modification and chromatin remodelling in ICC (28), and its mutation is strongly associated with loss of expression of the corresponding protein (29). The association identified between BAP1 mutation and HVEM expression in ICC progression may provide a new direction for study of the HVEM network in tumor immunity.

The present study has some limitations. Firstly, the small sample of data from a single institution may lead to false negative results in the statistical analysis. Secondly, the effects of HVEM blockade on immunity and tumor progression were not investigated in vitro and in vivo due to the lack of available HVEM inhibitors.

In conclusion, the present study demonstrated for the first time, to the best of our knowledge, that high HVEM expression was significantly associated with improved postoperative survival and low TNM stage in ICC, indicating that HVEM might be a favorable prognostic marker for ICC. Furthermore, the co-stimulatory signals from HVEM may play a dominant role in the progression of ICC, which may be explained by an increase in the number of PBLs rather than a change in the number of TILs. However, the function of the HVEM network in ICC progression is complex and requires further study.

\section{Acknowledgements}

Not applicable.

\section{Funding}

This research was supported by funding from National Natural Fund Project (grant no. 81572434), Ministry of Science and Technology, National Science and Technology Major Special Project: Prevention and Treatment of Major Infectious Diseases such as AIDS and Viral Hepatitis (grant no. 2018ZX10723204-007-001), 'Young Medical Elites', Tianjin Health Commission (duration of grant 2018.12-2020.11), 'Young Innovative Talents', Tianjin Medical University Cancer Institute and Hospital (duration of grant 2017.1-2019.12), Science and Technology Development Plan of Weifang in 2020 (Medical Science).

\section{Availability of data and materials}

The datasets used and/or analyzed during the current study are available from the corresponding author on reasonable request.

\section{Authors' contributions}

BM was responsible for study conception and design, data collection and analysis, performing experiments, writing the manuscript and statistical analysis. HM contributed to study conception and design, data analysis and writing the manuscript. YT performed data interpretation and provided technical and material support. YW, TS, TZ, QW, YC and HL performed data collection and data analysis. WZ and QL contributed to study conception, data collection and data interpretation, and critically revised the manuscript. All authors read and approved the final manuscript. 


\section{Ethics approval and consent to participate}

The Medical Ethics Committee of Tianjin Medical University Cancer Institute and Hospital approved the present study (approval no. bc2019065), and written informed consent was obtained from all patients or their legal guardian.

\section{Patient consent for publication}

Not applicable.

\section{Competing interests}

The authors declare that they have no competing interests.

\section{References}

1. Bergquist A and von Seth E: Epidemiology of cholangiocarcinoma. Best Pract Res Clin Gastroenterol 29: 221-232, 2015.

2. Mavros MN, Economopoulos KP, Alexiou VG and Pawlik TM: Treatment and prognosis for patients with intrahepatic cholangiocarcinoma: Systematic review and meta-analysis. JAMA Surg 149: 565-574, 2014.

3. Gonzalez-Carmona MA, Bolch M, Jansen C, Vogt A, Sampels M, Mohr RU, van Beekum K, Mahn R, Praktiknjo M, Nattermann J, et al: Combined photodynamic therapy with systemic chemotherapy for unresectable cholangiocarcinoma. Aliment Pharmacol Ther 49: 437-447, 2019.

4. Bridgewater J, Galle PR, Khan SA, Llovet JM, Park JW, Patel T, Pawlik TM and Gores GJ: Guidelines for the diagnosis and management of intrahepatic cholangiocarcinoma. J Hepatol 60: 1268-1289, 2014.

5. Rizvi S, Khan SA, Hallemeier CL, Kelley RK and Gores GJ: Cholangiocarcinoma-evolving concepts and therapeutic strategies. Nat Rev Clin Oncol 15: 95-111, 2018.

6. Martin-Liberal J, Ochoa de Olza M, Hierro C, Gros A, Rodon J and Tabernero J: The expanding role of immunotherapy. Cancer Treat Rev 54: 74-86, 2017.

7. Montgomery RI, Warner MS, Lum BJ and Spear PG: Herpes simplex virus-1 entry into cells mediated by a novel member of the TNF/NGF receptor family. Cell 87: 427-436, 1996.

8. Kwon BS, Tan KB, Ni J, Oh KO, Lee ZH, Kim KK, Kim YJ, Wang S, Gentz R, Yu GL, et al: A newly identified member of the tumor necrosis factor receptor superfamily with a wide tissue distribution and involvement in lymphocyte activation. J Biol Chem 272: 14272-14276, 1997.

9. Šedý JR and Ramezani-Rad P: HVEM network signaling in cancer. Adv Cancer Res 142: 145-186, 2019.

10. Ritthipichai K, Haymaker CL, Martinez M, Aschenbrenner A, YiX,Zhang M, Kale C, Vence LM, Roszik J,Hailemichael Y, et al: Multifaceted Role of BTLA in the control of CD8(+) T-cell Fate after Antigen Encounter. Clin Cancer Res 23: 6151-6164, 2017.

11. Sideras K, Biermann K, Yap K, Mancham S, Boor PPC, Hansen BE, Stoop HJA, Peppelenbosch MP, van Eijck CH, Sleijfer S, et al: Tumor cell expression of immune inhibitory molecules and tumor-infiltrating lymphocyte count predict cancer-specific survival in pancreatic and ampullary cancer. Int J Cancer 141: 572-582, 2017.

12. Hokuto D, Sho M, Yamato I, Yasuda S, Obara S, Nomi T and Nakajima Y: Clinical impact of herpesvirus entry mediator expression in human hepatocellular carcinoma. Eur J Cancer 51: $157-165,2015$.

13. Migita K, Sho M, Shimada K, Yasuda S, Yamato I, Takayama T, Matsumoto S, Wakatsuki K, Hotta K, Tanaka T, et al: Significant involvement of herpesvirus entry mediator in human esophageal squamous cell carcinoma. Cancer 120: 808-817, 2014.

14. Lan X, Li S, Gao H, Nanding A, Quan L, Yang C, Ding S and Xue Y: Increased BTLA and HVEM in gastric cancer are associated with progression and poor prognosis. Onco Targets Ther 10: 919-926, 2017.
15. Tang M, Cao X, Li Y, Li GQ, He QH, Li SJ, Chen J, Xu GL and Zhang KQ: High expression of herpes virus entry mediator is associated with poor prognosis in clear cell renal cell carcinoma. Am J Cancer Res 9: 975-987, 2019.

16. Zhang T, Ye L, Han L, He Q and Zhu J: Knockdown of HVEM, a lymphocyte regulator gene, in ovarian cancer cells increases sensitivity to activated T cells. Oncol Res 24: 189-196, 2016.

17. Fang Y, Ye L, Zhang T, He QZ and Zhu JL: High expression of herpesvirus entry mediator (HVEM) in ovarian serous adenocarcinoma tissue. J BUON 22: 80-86, 2017.

18. Tsang JYS, Chan KW, Ni YB, Hlaing T, Hu J, Chan SK, Cheung SY and Tse GM: Expression and clinical significance of herpes virus entry mediator (HVEM) in breast cancer. Ann Surg Oncol 24: 4042-4050, 2017.

19. Malissen N, Macagno N, Granjeaud S, Granier C, Moutardier V, Gaudy-Marqueste C, Habel N, Mandavit M, Guillot B, Pasero C, et al: HVEM has a broader expression than PD-L1 and constitutes a negative prognostic marker and potential treatment target for melanoma. Oncoimmunology 8: e1665976, 2019.

20. Ma B, Meng H, Tian Y, Wang Y, Song T, Zhang T, Wu Q, Cui Y, Li H, Zhang W and Li Q: Distinct clinical and prognostic implication of IDH1/2 mutation and other most frequent mutations in large duct and small duct subtypes of intrahepatic cholangiocarcinoma. BMC Cancer 20: 318, 2020.

21. Kang J, Jeong JH, Hwang HS, Lee SS, Park DH, Oh DW, Song TJ, Kim KH, Hwang S, Hwang DW, et al: Efficacy and Safety of Pembrolizumab in patients with refractory advanced biliary tract cancer: Tumor proportion score as a potential biomarker for response. Cancer Res Treat 52: 594-603, 2020.

22. Dobosz P, Stempor PA, Roszik J, Herman A, Layani A, Berger R, Avni D, Sidi Y and Leibowitz-Amit R: Checkpoint genes at the cancer side of the immunological synapse in bladder cancer. Transl Oncol 13: 193-200, 2020.

23. Tamada K, Shimozaki K, Chapoval AI, Zhu G, Sica G, Flies D, Boone T, Hsu H, Fu YX, Nagata S, et al: Modulation of T-cell-mediated immunity in tumor and graft-versus-host disease models through the LIGHT co-stimulatory pathway. Nat Med 6: 283-289, 2000 .

24. Zhao Q, Zhang GL, Zhu X, Su D, Huang ZL, Hu ZX and Peng L: The paradoxical changes of membrane and soluble herpes virus entry mediator in hepatocellular carcinoma patients. J Gastroenterol Hepatol 32: 1520-1524, 2017.

25. Liu J, Li J, He M, Zhang GL and Zhao Q: Distinct changes of BTLA and HVEM expressions in circulating $\mathrm{CD}^{+}$and $\mathrm{CD}^{+} \mathrm{T}$ cells in hepatocellular carcinoma patients. $\mathrm{J}$ Immunol Res 2018: 4561571, 2018

26. Komuta M, Govaere O, Vandecaveye V, Akiba J, Van Steenbergen W, Verslype C, Laleman W, Pirenne J, Aerts R, Yano $\mathrm{H}$, et al: Histological diversity in cholangiocellular carcinoma reflects the different cholangiocyte phenotypes. Hepatology 55: 1876-1888, 2012.

27. Liau JY, Tsai JH, Yuan RH, Chang CN, Lee HJ and Jeng YM: Morphological subclassification of intrahepatic cholangiocarcinoma: Etiological, clinicopathological, and molecular features. Mod Pathol 27: 1163-1173, 2014.

28. Jiao Y, Pawlik TM, Anders RA, Selaru FM, Streppel MM, Lucas DJ, Niknafs N, Guthrie VB, Maitra A, Argani P, et al: Exome sequencing identifies frequent inactivating mutations in BAP1, ARID1A and PBRM1 in intrahepatic cholangiocarcinomas. Nat Genet 45: 1470-1473, 2013.

29. Peña-Llopis S, Vega-Rubín-de-Celis S, Liao A, Leng N, Pavía-Jiménez A, Wang S, Yamasaki T, Zhrebker L, Sivanand S, Spence P, et al: BAP1 loss defines a new class of renal cell carcinoma. Nat Genet 44: 751-759, 2012

This work is licensed under a Creative Commons Attribution-NonCommercial-NoDerivatives 4.0 International (CC BY-NC-ND 4.0) License. 